\title{
EL ESTADO DE DERECHO ANTE LA VIOLENCIA INTRAFAMILIAR EN HERMOSILLO
}

\author{
Mireya Esthela GONZÁLEZ RODRÍGUEZ ${ }^{61}$ \\ Miguel Ángel LÓPEZ URIARTE ${ }^{62}$ \\ Jesús Miguel MAYA RODRÍGUEZ ${ }^{63}$
}

\begin{abstract}
SUMARIO: I. RESUMEN. II. INTRODUCCIÓN. III. JUSTIFICACIÓN. IV. ANTECEDENTES. V. MARCO LEGAL. VI. METODOLOGÍA. VII. RESULTADOS. VIII. CONCLUSIONES. VIII. BIBLIOGRAFÍA
\end{abstract}

\section{RESUMEN}

La violencia intrafamiliar, también conocida como doméstica, constituye una problemática social que hoy en día afecta cada vez más a los ciudadanos, y fue a partir del estudio llevado a cabo por INEGI $^{64}$ en el año 2003, que las autoridades e instituciones no gubernamentales trabajaron aportando sus propuestas e iniciativas que dieron como resultado la Ley General de Acceso a una Vida Libre de Violencia, así como algunos estudios de diagnóstico e investigación como el que se realizó en el Programa de Coinversión Social 2010, dentro de la convocatoria para "Prevenir y Reducir la Violencia en la Comunidad para el Fortalecimiento de la familia en Hermosillo, Sonora", con el proyecto "Discordancia en Violencia Intrafamiliar en Hermosillo, Sonora", que nos permitió realizar estudio mediante el cual se encontraron algunos datos importantes sobre el fenómeno en la comunidad que fueron detectados con el levantamiento de datos utilizando un instrumento adaptado denominado ENDIREH ${ }^{65}$, siendo aplicado bajo el diseño probabilístico aleatorio simple estratificado y por conglomerados bajo el muestreo de los hogares de la Colonia El Sahuaro en el Municipio de Hermosillo, Sonora, de los cuales se obtuvieron en algunos resultados incidencia de violencia intrafamiliar en un $44.2 \%$ de la población, de los cuales un $43.31 \%$ sufren violencia emocional, e intimidación en un $12.80 \%$.

\footnotetext{
${ }^{61}$ Profesora de Tiempo Completo Asociado "c", estudios de maestría en Políticas de Seguridad Pública, miembro de la Academia Jurídico-Formativa Departamento de Derecho, Universidad de Sonora, México.

62 Profesor de Tiempo Completo Asociado "c", estudios de maestría en Derecho Familiar, miembro de la Academia Jurídico-Formativa Departamento de Derecho, Universidad de Sonora, México.

${ }^{63}$ Licenciado en Derecho, Miembro Asociado Cuerpo Académico CA-UNISON-109 Universidad de Sonora, México, estudiante del programa Maestría en Innovación Educativa.

${ }^{64}$ Instituto Nacional de Estadística y Geografía.

${ }^{65}$ Encuesta Nacional sobre la Dinámica de las Relaciones en los Hogares (INEGI)
} 
Palabras Clave: Derecho, Violencia, intrafamiliar, comunitaria, investigación.

\section{INTRODUCCIÓN}

En la ciudad de Hermosillo, Sonora se ha generado un problema creciente en violencia social, esta se incrementado también dentro del seno familiar originando el problema de violencia intrafamiliar que aqueja a nuestra comunidad, y encontrando algunos índices alarmantes en nuestra entidad como lo fue el estudio llevado a cabo por INEGI en el año 2003, que especifica que en Sonora se encuentra en un $49.8 \%$ en violencia intrafamiliar apareciendo como el porcentaje más alto en violencia intrafamiliar en México, lo que originó que algunas instituciones y dependencias de gobierno llevaran a cabo diversos programas para prevenir el fenómeno como implementar la Ley General de acceso a una vida libre de violencia así como algunos estudios de diagnóstico e investigación para descifrar el problema que se ha extendido en varios estratos de la Sociedad Sonorense, entre estos estudios se encuentra el que se llevó a cabo por parte de la Universidad de Sonora con el Instituto Nacional de Desarrollo Social en el Programa de Coinversión Social 2010, dentro de la Convocatoria que se denominó PREVENCIÓN Y REDUCCIÓN DE LA VIOLENCIA EN LA COMUNIDAD PARA EL FORTALECIMIENTO DE LAS FAMILIAS EN HERMOSILLO, SONORA, con el proyecto DISCORDANCIA EN REGISTRO DE VIOLENCIA INTRAFAMILIAR EN HERMOSILLO, SONORA que nos permitió llevar a cabo diagnósticos de tipo sociodemográfico en la comunidad que pudieron ser cofactores de la situación de violencia intrafamiliar que se padece en los hogares de nuestra comunidad y cuyo análisis forma parte del trabajo que aquí se desarrolla.

\section{JUSTIFICACIÓN}

El aumento en violencia intrafamiliar como un fenómeno multifactorial que afecta a nuestra comunidad como lo muestra el estudio reciente del programa de Coinversión Social 2010 firmado por la Universidad de Sonora y el Instituto Nacional de Desarrollo Social en el que participo el H. Ayuntamiento de Hermosillo con la convocatoria denominada PREVENCIÓN Y REDUCCIÓN DE VIOLENCIA EN LA COMUNIDAD PARA EL FORTALECIMIENTO DE LAS FAMILIAS EN HERMOSILLO, SONORA, y los posibles daños que este problema ocasiona a las familias como un problema de salud integral, en el desarrollo de la economía y en la cultura propia de cada Estado, así como las condiciones desfavorables como pobreza, exclusión, marginación y desempleo fueron las directrices que seguí en

$$
\sim 76 \sim
$$


la ponencia que aquí se presenta y que constituyen una línea de investigación cuyos hallazgos en el análisis estadístico que se realizó pueden coadyuvar a llevar a cabo estudios de mayor profundidad y presupuesto que puedan incidir en el desarrollo de políticas públicas que beneficien a la sociedad por parte de las autoridades Federales, Estatales y Municipales.

\section{ANTECEDENTES}

En los años noventa, la violencia contra la mujer se constituyó en centro de atención e interés de las organizaciones internacionales. Como consecuencia de este interés se sucedieron hechos de gran importancia en el mundo que repercutieron a nivel nacional.(INEGI, 2003).En 1993, la Asamblea General de las Naciones Unidas aprobó la Declaraciones sobre la Eliminación de la violencia contra la mujer, la cual fue ratificada por México en 1995. En 1994, la Organización de los Estados Americanos (OEA) negoció la Convención Interamericana para Prevenir, Castigar y Erradicar la Violencia contra la Mujer, la cual fue ratificada por México en 1998.

En 1999, formulo el Protocolo Facultativo de la Convención para la Eliminación de todas las formas de Discriminación contra la mujer, el cual fue suscrito por México en ese año. Asimismo, el Fondo de Población de las Naciones Unidas, declaró que la violencia contra la mujer es "una prioridad de la salud pública" (INMUJERES, 2002).La Asamblea General de las Naciones Unidas declaró el 25 de Noviembre como día Internacional para la Eliminación de la Violencia contra las Mujeres, a través de la resolución 54/134 (INMUJERES, 2002).

La encuesta sobre Violencia Intrafamiliar (ENVIF), registró que uno de cada tres hogares del Área Metropolitana de la Ciudad de México sufre algún tipo de violencia intrafamiliar, asimismo, reveló una mayor presencia de actos de violencia en los hogares con jefatura masculina, $32.5 \%$ de estos hogares reporto algún tipo de $22 \%$ de los dirigidos por mujeres.

El marco legal establecido primero por el Comité para la eliminación de la discriminación contra la mujer (CEDAW) en 1979 con criterios mucho más específicos por la Convención Interamericana sobre la Prevención, Sanción y Erradicación de la Violencia contra las Mujeres (Belém do Pará, 1994), obliga a los gobiernos a implementar y dar seguimiento a leyes y políticas para sancionar la violencia contra las mujeres. Actualmente la mayoría de los países de la Región han ratificado las dos convenciones y cuentan con leyes sobre la violencia intrafamiliar/doméstica; algunas de las cuales hacen énfasis sobre la violencia contra la mujer. 
A pesar de estos avances en el diseño de políticas se han enfrentado limitaciones en relación con los contenidos y la implementación de las leyes. Por ejemplo, algunas legislaciones no tipifican determinados delitos como la violación conyugal, la violencia sexual en el hogar, ni el acoso sexual. (Organización Panamericana de la Salud OPS, 2003). Por otro lado, aunque la mediación o conciliación de casos de violencia intrafamiliar no es recomendada por las posturas legislativas y doctrinarias más modernas del derecho comparado ésta constituye uno de los medios más extendidos en la administración de justicia (Entidad de las Naciones Unidas para la Igualdad de Género y el Empoderamiento de la Mujer UNIFEM).

La Ley de Acceso de las mujeres a una vida libre de violencia.

"Artículo 4.- Los principios rectores para el acceso de todas las mujeres a una vida libre de violencia que deberán ser observados en la elaboración y ejecución de las políticas públicas federales y locales son:

I. La igualdad jurídica entre la mujer y el hombre;

II. El respeto a la dignidad humanas de las mujeres,

III. La no discriminación, y

IV. La libertad de las mujeres

Articulo 6.-Los tipos de violencia contra las mujeres son:

1. La violencia psicológica. Es cualquier acto u omisión que dañe la estabilidad psicológica, que puede consistir en; negligencia, abandono, descuido reiterado, celotipia, insultos, humillaciones, devaluación, marginación, indiferencia, infidelidad, comparaciones destructivas, rechazo, restricción a la autodeterminación y amenazas, las cuales conllevan a la víctima a la depresión, al aislamiento a la devaluación de su autoestima e incluso al suicidio;

2. La violencia física.-Es cualquier acto que inflige daño no accidental, usando la fuerza física o algún tipo de arma u objeto que pueda provocar o no lesiones ya sean internas, externas o ambas.

3. La violencia patrimonial.- Es cualquier acto u omisión que afecta la supervivencia de la víctima. Se manifiesta en la transformación, sustracción, retención o distracción de objetos, documentos personales, bienes y valores, derechos patrimoniales o recursos económicos destinados a satisfacer sus necesidades y puede abarcar los daños a los bienes comunes o propios de la víctima.

4. La violencia económica.- Es toda acción u omisión del agresor que afecta la supervivencia económica de la víctima. Se manifiesta a través de limitaciones encaminadas a controlar el ingreso de sus percepciones económicas, así como la percepción de un salario menor por igual trabajo, dentro de un mismo centro laboral.

5. La violencia sexual.- Es cualquier acto que degrada o daña el cuerpo y/o la sexualidad de la víctima y que por tanto atenta contra su libertad, dignidad e 
integridad física. Es una expresión de abuso de poder que implica la supremacía masculina sobre la mujer, al denigrarla y concebirla como objeto, y

6. Cualesquiera otras formas análogas que lesionen o sean susceptibles de dañar la dignidad, integridad o libertad de las mujeres."(Ley General de Acceso a una vida libre de violencia ,2007).

Los Programas de tratamiento en la Unión Europea.- En la guía de buenas prácticas para paliar los efectos de la violencia contra las mujeres editada en España en 2002, se apuntó que en trece Estados de la Unión Europea se disponía de medidas específicas de Rehabilitación de agresores. Y en once de ellos había tratamientos psicológicos o psiquiátricos, dentro de la prisión y solo en seis estados (Austria, Holanda, España, Irlanda, Reino Unido, y Suecia) se producía una participación en los programas de forma Sistemática (Díaz-Aguado y Martínez, 2002)

Informe de la Organización Mundial de la Salud.- Se han apuntado también otros factores comunitarios o sociales en relación con la ocurrencia de la violencia tal como se reconoce en su Informe la OMS, no se cuenta con una base empírica sólida. Entre los factores destacan: La existencia en la comunidad de una mayor tasa de otros delitos violentos, el capital social (en tanto que sociabilidad en la propia comunidad y la posibilidad de llevar a cabo una acción común), La existencia de normas relacionadas con la vida familiar y con la autoridad masculina sobre las mujeres (La existencia de desigualdades estructurales entre los hombres y las mujeres y de roles de género rígidos). Asimismo parecen ser factores sociales que pueden contribuir a una mayor tasa de violencia en aquellos lugares en los que el empleo de la violencia es común, se produce o se ha producido una confrontación bélica, <Las sociedades en las cuales los hombres tienen poder económico y de toma de decisiones en el hogar, donde las mujeres no tienen acceso fácil al divorcio y donde los adultos recurren habitualmente a la violencia para resolver sus conflictos> (Organización Mundial de la Salud, 2003).

\section{METODOLOGÍA}

Se utilizó una adaptación del instrumento ENDIREH (Encuesta Nacional de la Dinámica en las Relaciones de los Hogares), a las finalidades de la investigación y al contexto local. Fue aplicado bajo el diseño probabilístico, aleatorio simple estratificado, y por conglomerados.

El marco de muestreo fueron los hogares de la colonia El Sahuaro en el municipio de Hermosillo, informados por INEGI (Instituto Nacional de 
Estadística Geografía e Informática) en el Censo de Población y Vivienda 2000.

La población estuvo formada por 3,241 hogares, teniéndose una muestra de 344 hogares, bajo un $95 \%$ de nivel de confianza y un $+/-5 \%$ de error muestral. El levantamiento se realizó del 25 de octubre al 15 de Noviembre de 2010.

El instrumento se aplicó a 344 hogares constituidos en la colonia El Sahuaro del Municipio de Hermosillo, aleatoriamente a la persona que abriera la puerta madre o padre de familia, el instrumento se conformó de datos de identificación de la vivienda, datos del entrevistado y módulos como hogar actual, sexualidad de la pareja, maltrato emocional, intimidación, abuso físico, abuso sexual y ayuda buscada.

Para el procesamiento de la información se alimentó una base de datos en el programa informático Statistical Package for the Social Sciences (SPSS) versión 18 conocido también como Predictiva Analytics SoftWare (PASW).La información recibió tratamiento estadístico en medidas de centralización.

\section{RESULTADOS}

Entre los datos obtenidos se encontraron los siguientes:

\begin{tabular}{|c|c|}
\hline \multicolumn{2}{|c|}{ Sexo } \\
\hline Masculino & Femenino \\
\hline $19.5 \%$ & $80.5 \%$ \\
\hline
\end{tabular}

Tabla 1 Sexo.

El $19.5 \%$ de los informantes fueron de sexo masculino y un $80.5 \%$ femenino.

\begin{tabular}{|c|c|c|c|c|c|}
\hline \multicolumn{7}{|c|}{ Edad } \\
\hline $16-25$ & $26-35$ & $36-45$ & $46-59$ & 60 y mas & N/C \\
\hline $19.5 \%$ & $80.5 \%$ & $22.4 \%$ & $33.7 \%$ & $18.6 \%$ & $2.9 \%$ \\
\hline
\end{tabular}

Tabla 2 Edad.

En cuanto a la edad el $80.5 \%$ de los informantes manifestaron tener al momento del levantamiento de datos entre $26-35$ años, seguido de un $33.7 \%$ en el rango de los 46-59 años de edad. 


\begin{tabular}{|c|c|c|}
\hline \multicolumn{3}{|c|}{ Estado Civil } \\
\hline Casado & Unión libre & NC \\
\hline $66.3 \%$ & $17.2 \%$ & $16.6 \%$ \\
\hline
\end{tabular}

Tabla 3 Estado Civil.

El $66.3 \%$ de los informantes manifestaron encontrarse en estado civil casado, un $17.2 \%$ en unión libre y un $16.6 \%$ no contesto.

\begin{tabular}{|c|c|c|c|c|c|}
\hline \multicolumn{5}{|c|}{ Ocupación } \\
\hline Trabajador & Estudiante & $\begin{array}{c}\text { Trabajador y } \\
\text { Estudiante }\end{array}$ & $\begin{array}{c}\text { Atiende el } \\
\text { Hogar }\end{array}$ & $\begin{array}{c}\text { Trabaja } \\
y \\
\text { atiende } \\
\text { el hogar }\end{array}$ & NC \\
\hline $12.8 \%$ & $0.6 \%$ & $4.7 \%$ & $57.6 \%$ & $18.3 \%$ & $6.1 \%$ \\
\hline
\end{tabular}

Tabla 4 Ocupación.

En cuanto a la ocupación que desempeñan los informantes el 57.6\% manifestó que únicamente atiende el hogar, el 18.3\% además de atender el hogar trabaja, el $12.8 \%$ solo trabaja, el $0.6 \%$ solo se dedica a los estudios y un $6.1 \%$ no contesto.

\begin{tabular}{|c|c|c|c|c|c|}
\hline \multicolumn{7}{|c|}{ Escolaridad } \\
\hline Ninguna & Primaria & Secundaria & Bachillerato & Profesional & NC \\
\hline $6.1 \%$ & $34.3 \%$ & $33.7 \%$ & $16.3 \%$ & $6.4 \%$ & $3.2 \%$ \\
\hline
\end{tabular}

Tabla 5 Escolaridad.

El 33.7\% de los informantes cuentan con educación secundaria, 34.3\% solo primaria, y un $16.3 \%$ bachillerato.

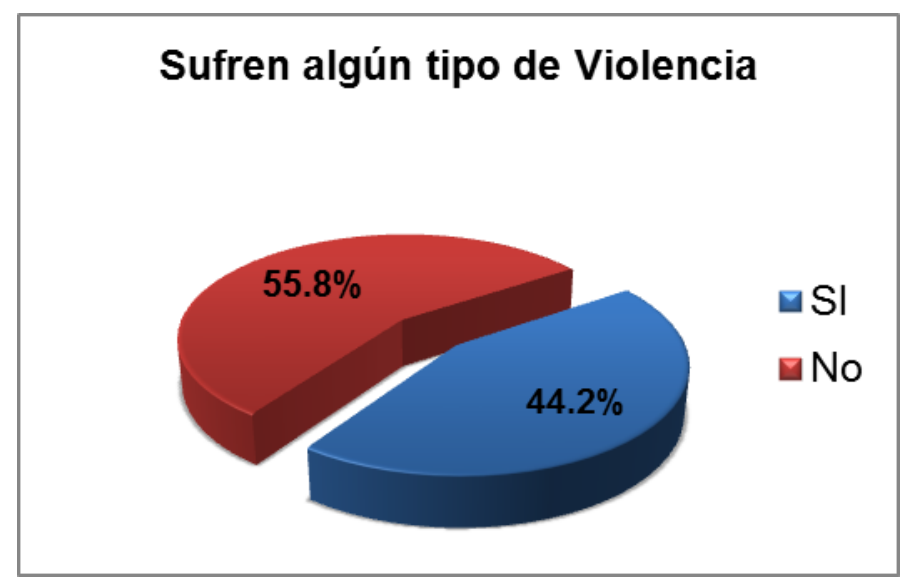

Gráfica 1 Incidencia global de violencia. 
El 44.2\% de los informantes sufren episodios de violencia al interior de los hogares, que se pueden derivar en actos de violencia emocional, intimidación, abuso físico, o abuso sexual.

\begin{tabular}{|c|c|c|c|c|}
\hline & \multicolumn{4}{|c|}{ Incidencia de Violencia } \\
\hline & Emocional & Intimidación & Abuso físico & Abuso sexual \\
\hline $\mathrm{Si}$ & $43.31 \%$ & $12.80 \%$ & $6.98 \%$ & $1.70 \%$ \\
\hline No & $56.69 \%$ & $87.20 \%$ & $93.02 \%$ & $98.30 \%$ \\
\hline
\end{tabular}

Tabla 6 Incidencia especifica de violencia.

En el caso de explorar la violencia especifica por tipo, el $43.31 \%$ de los informantes manifiestan haber vivido algún episodio de violencia emocional, un $12.80 \%$ algún tipo de violencia por intimidación, el $6.98 \%$ de abuso físico, y un $1.70 \%$ algún tipo de violencia sexual.

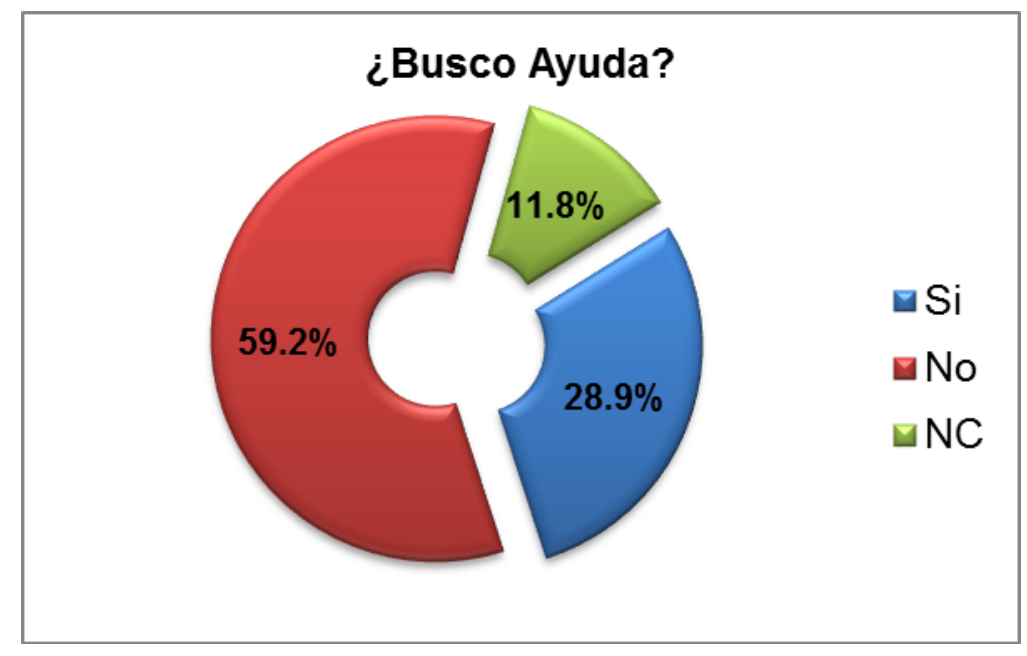

\section{Gráfica 2 Busco ayuda.}

Solo el $28.9 \%$ de los informantes manifestaron haber buscado algún tipo de ayuda para resolver la situación de violencia doméstica. 


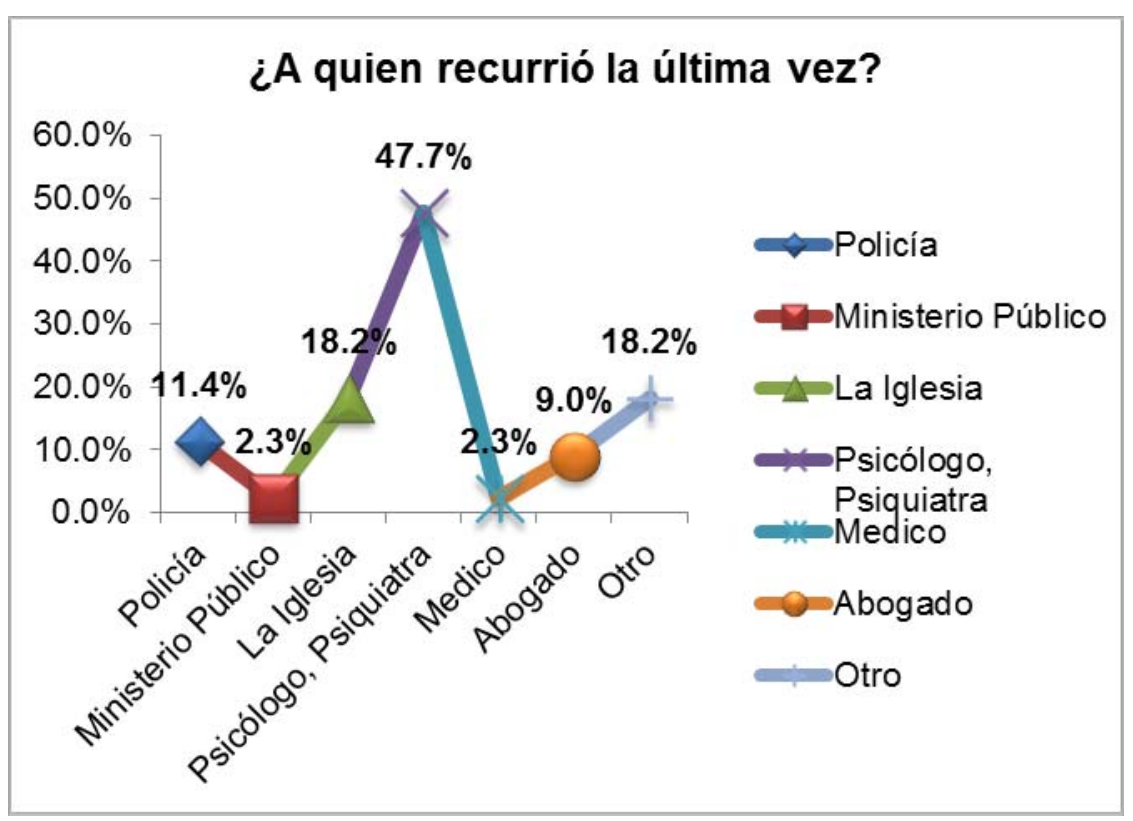

\section{Gráfica 3. A quien recurrió en la búsqueda de ayuda}

De los informantes que recurrieron a ayuda, el $47.7 \%$ opto por los servicios de psicólogo o psiquiatra, en un $18.2 \%$ a la iglesia, $11.4 \%$ solicitaron el auxilio de la fuerza publica por medio de la policia, el $9.0 \%$ apelaron a los servicios de un abogado, y en igual porcentaje $2.3 \%$ acudieron al ministerio público o el medico, un $18.2 \%$ manifesto solicitar ayuda en otras formas.

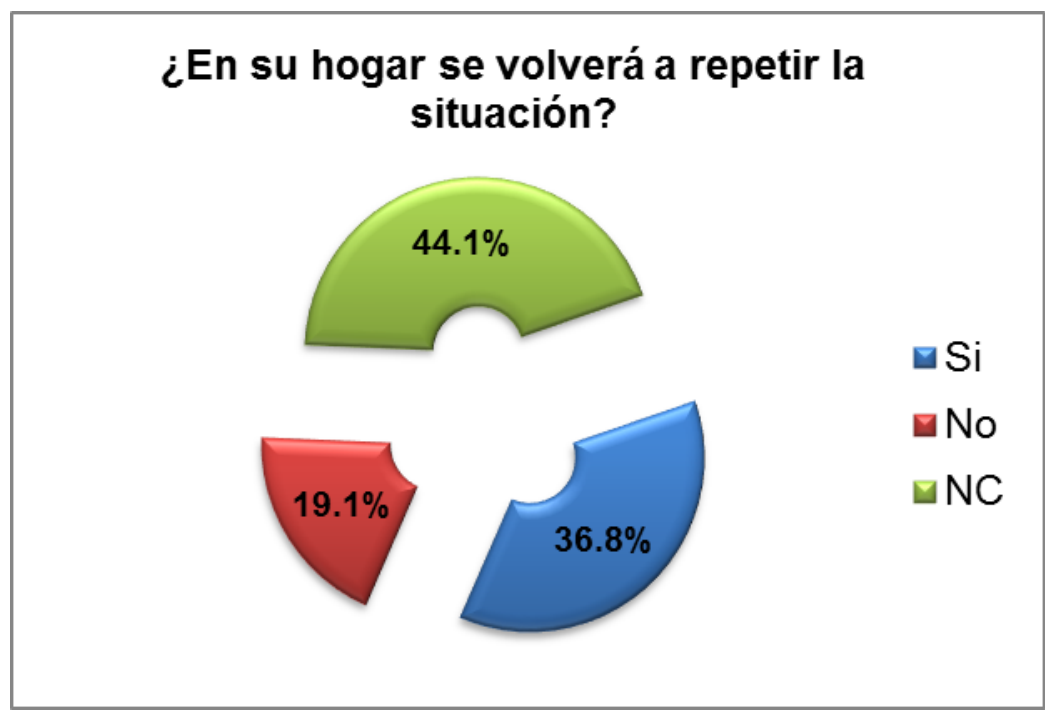

Gráfica 4. Proyección de la muestra de nuevos eventos violentos. 


\section{CONCLUSIONES}

1.-El hecho de que dentro de este estudio el $55.8 \%$ de las familias sufren violencia nos indica que existe un problema serio en nuestra comunidad el cual no debe ser minimizado y tomarse medidas serias que vayan encaminadas no solo para penalizar o endurecer el aspecto lega sino que los órganos competentes de los Estados se den cuenta que es un fenómeno que va ligado a aspectos sociales, económicos y culturales y que debemos trabajar en torno a Políticas Públicas para erradicar el problema.

2.-El $36.8 \%$ de las personas que respondieron la encuesta realizada estuvieron de acuerdo en que en su hogar volvería a repetirse una situación de violencia, lo que refleja la constante de violencia intrafamiliar que forma parte de sus interacciones cotidianas dentro del seno familiar, aun cuando en cada familia se varíe en la intensidad de su problemática.

3.- El $47.7 \%$ de los encuestados manifestaron haber acudido al médico y al psicólogo lo que puede ser indicador de que las personas se sientan enfermas o tengan conocimiento que la violencia intrafamiliar que padecen es como lo está indicando el presente estudio un fenómeno multijcausal que también está afectando su salud entre otros factores.

4.-El mayor índice de la población encuestada tiene alrededor de 46-59 años lo que nos indica entre otras cosas que es una comunidad adulta y que por las condiciones de muy baja escolaridad, recursos económicos muy precarios, viviendas abandonadas, falta de servicios básicos como pavimentación y drenaje, espacios públicos para esparcimiento, vandalismo etc.requieren de apoyo de las autoridades y demás organismos para tener acceso a una mejor calidad de vida.

\section{BIBLIOGRAFÍA}

Instituto Nacional de Estadística y Geografía, (2003). "Estadísticas a propósito del día internacional para la eliminación de la violencia contra las mujeres". INEGI, México, 2003, Consultado en: http://www.culturadelalegalidad.org.mx/recursos/Contenidos/Estadsticas/do cumentos/Violencia\%20intrafamiliar.pdf. 
INMUJERES (2002), "Breviarios Informativos de Género". INAM, INMUJERES, México, 2002, pp. 1-5 y 5-18.

Belém do Pará., Convención interamericana para prevenir sancionar y erradicar la violencia contra la mujer, convención de belém do pará, Brasil, 1994 Consultado en: http://www.cidh.org/Basicos/Spanish/Basicos6.htm.

Organización Panamericana de la Salud, marco de acción en salud ambiental OPS 2003-2007, USA, 2003 Consultado en: http://www.bvsde.paho.org/bvsadi/fulltext/marco.pdf.

United Nations Development Fund for Women, Convención sobre la Eliminación de todas las formas de Discriminación contra la Mujer. USA, 1995, Consultado en: http://www.un.org/ga/search/view doc.asp?symbol=A/RES/34/180\&Lang=S

DÍAZ-AGUADO, M. J.; MARTÍNEZ ARIAS, R., "Guía de Buenas Prácticas para paliar los efectos de la violencia contra las mujeres y conseguir su erradicación", Presidencia de la Unión Europea, Madrid, 2002, Consultado en: www.mtas.es/mujer/violpresi.htp

Organización Mundial de la Salud, Informe sobre la salud en el mundo, OMS, Suiza 2003, Consultado

en: http://libdoc.who.int/hq/2003/WHO WHR 03.1 spa.pdf. 